
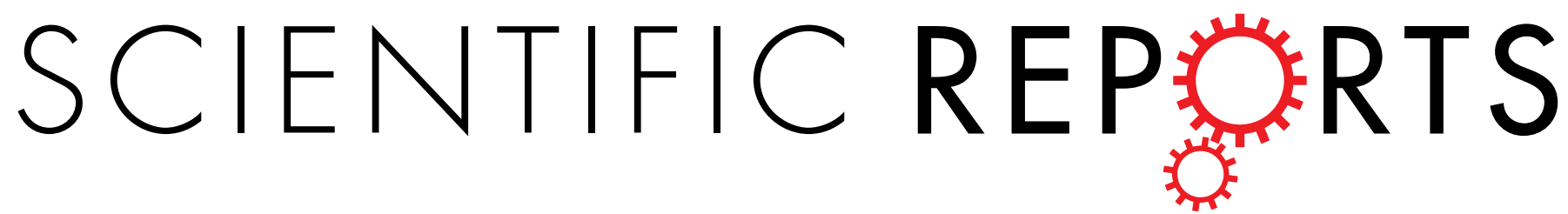

\title{
OPEN
}

Received: 14 December 2017

Accepted: 17 July 2018

Published online: 27 July 2018

\section{Prenatal Bisphenol A Exposure is Linked to Epigenetic Changes in Glutamate Receptor Subunit Gene Grin2b in Female Rats and Humans}

\author{
Ali Alavian-Ghavanini ${ }^{1,2}$, Ping-I Lin ${ }^{3}$, P. Monica Lind ${ }^{4}$, Sabina Risén Rimfors ${ }^{1}$, Margareta Halin \\ Lejonklou ${ }^{4}$, Linda Dunder ${ }^{4}$, Mandy Tang ${ }^{1}$, Christian Lindh ${ }^{5}$, Carl-Gustaf Bornehag ${ }^{3,6}$ \& \\ Joëlle Rüegg $\mathbb{1}^{1,2}$
}

Bisphenol A (BPA) exposure has been linked to neurodevelopmental disorders and to effects on epigenetic regulation, such as DNA methylation, at genes involved in brain function. High doses of BPA have been shown to change expression and regulation of one such gene, Grin2b, in mice. Yet, if such changes occur at relevant doses in animals and humans has not been addressed. We investigated if lowdose developmental BPA exposure affects DNA methylation and expression of $G$ rin2 $b$ in brains of adult rats. Furthermore, we assessed associations between prenatal BPA exposure and Grin $2 b$ methylation in 7-year old children. We found that Grin2b mRNA expression was increased and DNA methylation decreased in female, but not in male rats. In humans, prenatal BPA exposure was associated with increased methylation levels in girls. Additionally, low APGAR scores, a predictor for increased risk for neurodevelopmental diseases, were associated with higher Grin $2 b$ methylation levels in girls. Thus, we could link developmental BPA exposure and low APGAR scores to changes in the epigenetic regulation of Grin2b, a gene important for neuronal function, in a sexual dimorphic fashion. Discrepancies in exact locations and directions of the DNA methylation change might reflect differences between species, analysed tissues, exposure level and/or timing.

Bisphenol A (BPA) is a widely used chemical in the production of polycarbonate plastics and epoxy resins existing in many common consumers and household products ${ }^{1}$. Consequently, humans are exposed to low levels of BPA on a daily basis. For instance, a population-based study in the U.S. showed that over $92 \%$ of children over the age of 6 had detectable levels of BPA in their urine ${ }^{2}$. Estimated BPA exposure levels are $0.01-0.4 \mu \mathrm{g} / \mathrm{kg}$ body weight (bw)/day for adults and $0.1-0.5 \mu \mathrm{g} / \mathrm{kg} \mathrm{bw} /$ day for children and adolescents ${ }^{3}$.

Numerous studies have demonstrated that BPA interferes with estrogen signalling and other hormonal signalling pathways, both in vitro and in animals, thus acting as an endocrine disrupting chemical (EDC) ${ }^{4-6}$. BPA targets, such as the estrogen system, are crucially involved in neurodevelopment, in particular in sexual differentiation of the brain ${ }^{7,8}$. Thus, interference with these systems during sensitive developmental periods could ultimately affect brain function and behaviour. Indeed, a number of epidemiological and experimental studies suggest that early life exposure to BPA might lead to behavioural alteration later in life ${ }^{6,9-12}$. In the majority of studies, the effects of BPA were sex-specific, often blunting or abolishing natural sexual dimorphisms ${ }^{12,13}$.

It is also known that BPA induces epigenetic changes, which are thought to underlie persistent effects of early life exposures ${ }^{13-16}$. Epigenetic processes drive cell differentiation by regulating long-term gene function, and are crucial for foetal development, including the brain ${ }^{17,18}$. Epigenetic marks regulate gene transcription in different ways. E.g., DNA methylation at transcription factor (TF) binding sites in regulatory regions can prevent TF

\footnotetext{
${ }^{1}$ Swetox, Karolinska Institutet, Unit of Toxicology Sciences, Forskargatan 20, 151 36, Södertälje, Sweden. ${ }^{2}$ Karolinska Institutet, Department of Clinical Neuroscience, Centre for Molecular Medicine (CMM), 171 64, Solna, Sweden. ${ }^{3}$ Karlstad University, Department of Health Sciences, 651 88, Karlstad, Sweden. ${ }^{4}$ Uppsala University, Department of Medical Sciences, Occupational and Environmental Medicine, 751 85, Uppsala, Sweden. ${ }^{5}$ Lund University, Division of Occupational and Environmental Medicine, Lund University, 221 85, Lund, Sweden. ${ }^{6}$ Icahn School of Medicine at Mount Sinai, New York, NY, USA. Ali Alavian-Ghavanini and Ping-I Lin contributed equally to this work. Correspondence and requests for materials should be addressed to J.R. (email: joelle.ruegg@swetox.se)
} 
binding and thus decrease gene transcription. There is ample evidence that BPA induces epigenetic changes in a variety of experimental systems, and several epidemiological studies indicate a link between epigenetic changes, in particular DNA methylation alterations, and chemical exposures including BPA ${ }^{16,19}$. In animal studies, BPA has been shown to affect DNA methylation of genes involved in brain development and function, such as $B d n f$, Fkbp5, and Grin $2 b^{20-22}$.

Grin $2 b$ is a particularly interesting target as genetic polymorphisms in this gene have been consistently found to be associated with neurodevelopmental diseases/disorders, such as Attention Deficit Hyperactivity Disorder (ADHD), Autism Spectrum Disorder (ASD), and schizophrenia ${ }^{23-25}$. The Grin2b gene encodes the NR2B subunit of N-methyl-D-aspartate receptors (NMDARs), which are receptors for the excitatory neurotransmitter glutamate and important for regulation of neural morphology, learning and memory ${ }^{26}$. Its transcription is, among others, regulated by the TF nuclear respiratory factor $1(\mathrm{Nrf1})^{27}$, whose DNA binding, in turn, is inhibited by DNA methylation ${ }^{28}$.

Glutamate receptor genetic functional variations may hold the key to glutamate status ${ }^{24}$, which could underlie their association with psychiatric disorders. For example, higher glutamate concentrations have been found in patients with schizophrenia ${ }^{29,30}$. Intriguingly, perinatal exposure to BPA has also been suggested to cause an increase in glutamate concentration in the rat hippocampus ${ }^{31}$. However, it remains unclear if changes in NMDAR composition contributes to this increase, and if epigenetic dysregulation of their subunit genes is involved. Furthermore, a link between BPA exposure and Grin $2 b$ expression or methylation has, to our knowledge, never been addressed in humans.

Here, we assessed whether developmental exposure to BPA in doses around or below the human tolerable daily intake set by the European Food Safety Authority (EFSA) $(4 \mu \mathrm{g} / \mathrm{kg}$ bw/day) and the U.S. Food and Drug Administration (FDA) $(50 \mu \mathrm{g} / \mathrm{kg}$ bw/day) persistently changes expression and methylation of Grin $2 b$ in Fischer 344 rat brain. Furthermore, we explored if prenatal BPA exposure is associated with DNA methylation of GRIN2B in 7-year old children, when adjusting for potential confounders.

\section{Results}

Effects of BPA on Grin2b in rats. In order to identify persistent changes in Grin $2 b$ upon developmental BPA treatment, DNA methylation and gene expression were analysed in 52 weeks old Fischer 344 rats. We used hippocampus for these analyses as this is a region expressing Grin $2 b$ even in the adult brain ${ }^{32}$. Dams were exposed to 0.5 or $50 \mu \mathrm{g} \mathrm{BPA} / \mathrm{kg}$ bw/day or vehicle (control) during gestation and lactation via their drinking water. No differences in body weight and other gross health parameters were observed between the treatment groups (see Supplementary Table S1). Analysis of hippocampal Grin $2 b$ expression showed that both doses of BPA lead to a significant increase in Grin $2 b$ expression in females $(\mathrm{p}=0.0094$ control vs. $0.5 \mu \mathrm{g}$ and $\mathrm{p}=0.0033$ control vs. $50 \mu \mathrm{g}$ ) whereas no significant change in males was detected, although a trend towards decreased expression was observed (Fig. 1a). This opposing effect of BPA in females vs. males led to elimination of a significant sex difference in Grin $2 b$ expression ( $\mathrm{p}<0.0001$ ) observed in the control animals.

To explore the role of epigenetic regulation for this change in Grin2b expression, we analysed DNA methylation at two adjacent regions in the Grin $2 b$ promoter, one containing a predicted Nrf1 binding site and one that is homologous to a region in mice that has previously been shown to be affected by BPA (depicted in Fig. 1b) ${ }^{22}$. DNA methylation analysis, performed in two independent runs, showed that prenatal BPA exposure at both doses leads to a significant decrease in methylation in females in the three CpGs that are part of the Nrf1 binding site $(\mathrm{CpG} 1: \mathrm{p}=0.019$ control vs. $0.5 \mu \mathrm{g}$ and $\mathrm{p}=0.036$ control vs. $50 \mu \mathrm{g} ; \mathrm{CpG} 2 \mathrm{p}=0.011$ control vs. $0.5 \mu \mathrm{g}$ and $\mathrm{p}=0.03$ control vs. $50 \mu$ g; CpG3: $p=0.053$ control vs. $0.5 \mu \mathrm{g}$ and $\mathrm{p}=0.035$ control vs. $50 \mu \mathrm{g}$ ). No significant changes were observed in males (Fig. 1c). For the CpGs in the adjacent region (rCpGs 5-7, homologous to the ones analysed $i^{22}$ ), no significant methylation differences were found in neither of the sexes (see Supplementary Fig. S1). These results did not change when normalised for potential batch effects using rank-based inverse transformation of the methylation data. Grin $2 b$ expression and methylation at CpG1-3 in females was inversely correlated (CpG1: $p=0.077 ; C p G 2: p=0.044 ; C p G 3: p=0.048$ ) (Fig. $1 d$ ), indicating that methylation at these CpGs is functionally involved in gene expression. No significant correlation was found between Grin2b expression and methylation at all analysed CpGs in males (see Supplementary Fig. S1).

Developmental exposure to BPA has been shown to affect growth ${ }^{33}$. To exclude that the observed effects are a result of growth differences between the treatment groups, Grin $2 b$ expression and methylation was adjusted to the animals' growth rates. Yet, the increase in Grin $2 b$ expression and decrease in DNA methylation in female rats in the treatment groups remained significant (see Supplementary Fig. S1).

Taken together, our data suggest that developmental exposure to BPA changes the DNA methylation level in the Grin $2 b$ promoter, resulting in altered gene expression levels in female, but not in male, rats one year after the exposure had ceased.

Prenatal BPA levels and GRIN2B methylation in children. Encouraged by the findings from the rat model, we attempted to assess if prenatal BPA exposure levels were associated with methylation level of the GRIN2B gene also in humans, in a subpopulation of the SELMA study ${ }^{34}$ (Table 1). Creatinine adjusted BPA concentrations were measured in the $1^{\text {st }}$ trimester urine in pregnant mothers. Based on these measurements, the daily intake of BPA for mothers of boys and girls were estimated to be $0.007-0.2 \mu \mathrm{g} / \mathrm{kg} / \mathrm{day}$ and $0.004-0.6 \mu \mathrm{g} / \mathrm{kg} / \mathrm{day}$, respectively (no significant difference between the two groups of mothers was noticed). Thus, the highest intake measured in the human cohort corresponded to the lower dose given in the rat study. GRIN2B methylation was assessed at $3 \mathrm{CpG}$ sites in a region homologous to the investigated rat locus (Fig. 1b), using buccal DNA from 7 year-old children. We identified 4 DNA methylation clusters since the $\mathrm{CH}$ pseudo-F value abruptly increased at this number of clusters for all CpG sites (see Supplementary Fig. S3). The methylation levels in each cluster are 
a

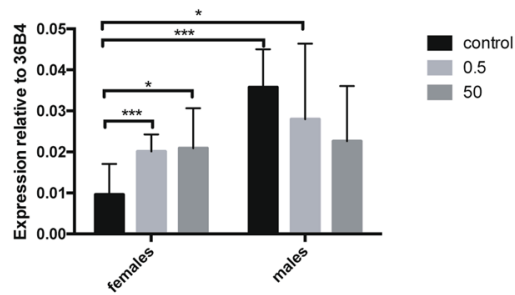

b

NRF1

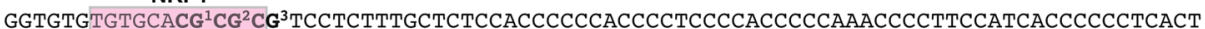
CCCACTGCTAAGACTCTTGGCTGAGGCTGCTTCTCACG ${ }^{4}$ TCATGAGCTGGGGGAAGTGGGGTAGCCAGGGGGTGGGAAAAGCTTA

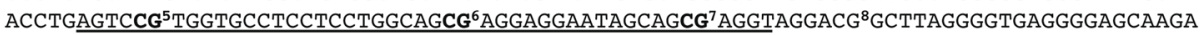
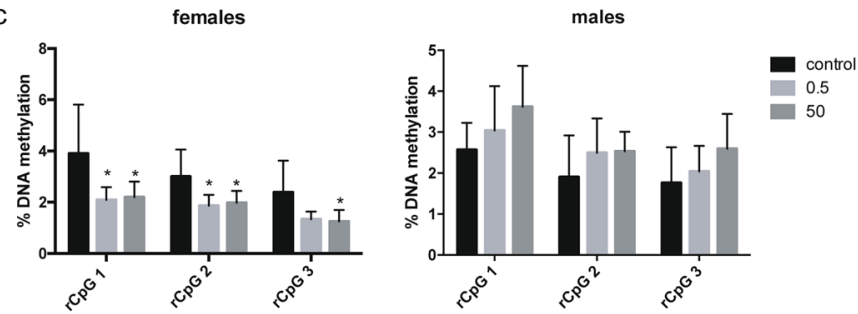

d
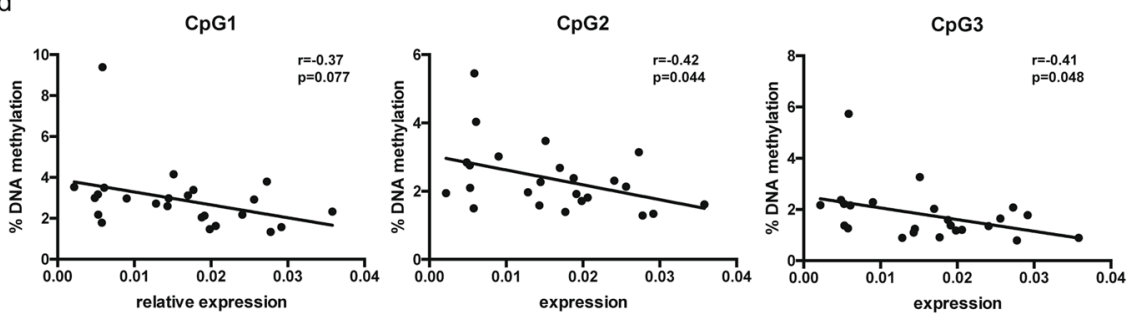

Figure 1. Developmental BPA exposure leads to changes in Grin2b expression and methylation in hippocampus of female rats one year after exposure had ceased. (a) Gene expression analysis of Grin $2 b$ assessed by qPCR in hippocampus of 52 weeks old rats developmentally exposed to $0.5 \mathrm{or} 50 \mu \mathrm{g} / \mathrm{kg}$ bw/day $\mathrm{BPA}$ or vehicle control. Bars show mean and standard deviation of Grin $2 b$ expression relative to the control gene 36B4 for female (control: $n=10,0.5: n=7,50: n=8)$ and male (control: $n=12,0.5: n=8,50: n=7$ ) animals. (b) Sequence of the two adjacent regions (underlined) analysed for DNA methylation in the rat Grin $2 b$ promoter. The predicted Nrf1 binding site is marked with a red box. (c) DNA methylation analysis of 3 CpGs in the promoter region of Grin $2 b$ assessed by bisulfite pyrosequencing in hippocampus of 52 weeks old rats developmentally exposed to 0.5 or $50 \mu \mathrm{g} / \mathrm{kg}$ bw/day BPA or vehicle control. Bars show mean and standard deviation of \% methylation for female (control: $\mathrm{n}=12,0.5: \mathrm{n}=8,50: \mathrm{n}=8$ ) and male (control: $\mathrm{n}=8,0.5: \mathrm{n}=8$, 50: $\mathrm{n}=8$ ) animals. (d) Correlation between relative Grin2b expression and methylation at CpG 1-3 in female rat hippocampus. ${ }^{*} \mathrm{p}<0.05, * * \mathrm{p}<0.01, * * * \mathrm{p}<0.001$ treatment animals compared to controls.

presented in Supplementary Table S2. Subsequent analyses were conducted separately for females and males to evaluate sex-specific differences.

Ordered logistic regression analysis results showed a significant association $(\mathrm{p}=0.049$ and $\mathrm{p}=0.039$ treating BPA quartile as an ordered categorical variable and dummy variable, respectively) between the methylation level of the first CpG site (denoted as hCpG1) and prenatal BPA levels (expressed both in a continuous scale as well as in quartiles and adjusted for creatinine) in female subjects, after adjusting for potential confounders (Table 2). As shown in Fig. 2a, BPA levels were positively correlated with hCpG1 methylations levels in girls but not in boys.

Prenatal BPA levels, APGAR scores, and GRIN2B methylation. We also noted that methylation levels at hCpG2 were significantly inversely correlated with APGAR scores, an index for infant's cardiovascular and pulmonary functions outside the womb (odds ratio $=0.21, p=0.007$ ) (Table 2). To assess the relationship between BPA and APGAR scores to predict GRIN2B methylation at hCpG1 and hCpG2, we used SEM, selecting methylation levels at these two sites as the two primary outcomes, while prenatal BPA levels and APGAR scores were treated as two primary predictors. We also evaluated if APGAR scores could serve as a mediator for the BPA effect on GRIN2B methylation. APGAR scores were significantly associated with the methylation levels at both CpG sites $(p=0.019$ for $h C p G 1$ and $p=0.012$ for $h C p G 2)$, whereas prenatal BPA levels were only significantly associated with methylation levels at hCpG1 $(\mathrm{p}=0.027)($ Fig. $2 \mathrm{~b})$, implying that APGAR score and prenatal BPA exposure act as two independent predictors for the methylation at hCpG1 of GRIN2B in girls. 


\begin{tabular}{|l|l|l|}
\hline Sample Characteristics & Females & Males \\
\hline Number of subjects N (\%) & $152(47.8)$ & $165(51.9)$ \\
\hline CpG1 (\%) Mean (SD) & $2.83(3.96)$ & $3.54(4.26)$ \\
\hline CpG2 (\%) Mean (SD) & $2.69(4.48)$ & $3.09(4.29)$ \\
\hline CpG2 (\%) Mean (SD) & $1.13(2.63)$ & $0.87(2.09)$ \\
\hline Bisphenol A (ng/mL) Geometric mean (95\% CI) & $1.67(1.47-1.89)$ & $1.62(1.41-1.87)$ \\
\hline Week of enrolment Mean (SD) & $9.95(2.11)$ & $10.2(2.45)$ \\
\hline 5 minutes APGAR score N (\%) & $134(88.2)$ & $145(87.9)$ \\
\hline Healthy (score = 10) & $18(11.8)$ & $20(12.1)$ \\
\hline Complication (score $<10)$ & $127(83.6)$ & $138(83.6)$ \\
\hline Maternal smoking status during pregnancy N (\%) & $12(7.9)$ & $12(7.3)$ \\
\hline No & $8(5.3)$ & $11(6.7)$ \\
\hline Passive & $128(84.2)$ & $148(89.7)$ \\
\hline Active & $24(15.8)$ & $17(10.3)$ \\
\hline Stressful events during pregnancy N (\%) & \\
\hline No & \multicolumn{2}{|l}{} \\
\hline Yes &
\end{tabular}

Table 1. Characteristics of 317 children from the SELMA pregnancy cohort. BPA values were adjusted for creatinine.

\begin{tabular}{|c|c|c|c|c|}
\hline & & All subjects & Females only & Males only \\
\hline \multirow{5}{*}{ Model 1} & Predictors & OR $(95 \% \mathrm{CI})$ & OR $(95 \% \mathrm{CI})$ & OR $(95 \% \mathrm{CI})$ \\
\hline & $\mathrm{BPA}^{\varphi}$ (quartile was treated as an ordered categorical variable) & $1.11(0.91-1.35)$ & $1.36(1.02-1.91)^{*}$ & $0.93(0.71-1.20)$ \\
\hline & APGAR score (5th minute; 10 vs. $<10)$ & $0.61(0.30-1.25)$ & $0.27(0.09-0.80)^{*}$ & $1.52(0.56-4.11)$ \\
\hline & Maternal smoking & $0.68(0.44-1.06)$ & $0.79(0.40-1.56)$ & $0.55(0.30-1.02)$ \\
\hline & Maternal stress & $1.20(0.65-2.22)$ & $0.75(0.31-1.82)$ & $2.39(0.95-6.03)$ \\
\hline \multirow{7}{*}{ Model 2} & BPA quartile (dummy variable) & & & \\
\hline & 2nd quartile vs. 1st quartile & $0.93(0.50-1.74)$ & $0.95(0.35-2.52)$ & $0.78(0.33-1.83)$ \\
\hline & 3rd quartile vs. 1st quartile & $0.73(0.39-1.37)$ & $0.78(0.28-2.15)$ & $0.59(0.25-1.36)$ \\
\hline & 4th quartile vs. 1st quartile & $1.50(0.82-2.76)$ & $2.72(1.05-7.01)^{*}$ & $0.85(0.37-1.93)$ \\
\hline & APGAR score (5th minute; 10 vs. $<10$ ) & $0.56(0.27-1.15)$ & $0.21(0.07-0.65)^{* *}$ & $1.49(0.55-4.06)$ \\
\hline & Maternal smoking & $0.68(0.43-1.07)$ & $0.84(0.42-1.67)$ & $0.53(0.29-0.99)^{*}$ \\
\hline & Maternal stress & $1.23(0.66-2.29)$ & $0.78(0.32-1.92)$ & $2.40(0.92-6.10)$ \\
\hline
\end{tabular}

Table 2. Association between prenatal BPA exposure and GRIN2B methylation, adjusted for potential confounders in each ordered logistic regression, expressed as OR (95\% CI). *p-value $<0.05$, **p-value $<0.01$. ${ }^{\varphi} \mathrm{BPA}$ values were adjusted for creatinine before they were assigned into a particular quartile.

\section{Discussion}

In the current study, we assessed effects of early life BPA exposure on methylation levels of the Grin $2 b$ gene in brain tissue from rats and buccal samples from humans. In the rat hippocampus, we observed that developmental BPA exposure lead to significantly decreased methylation levels in Grin $2 b$ in females, but not in males. Furthermore, decreased methylation in female rats was concomitant with increased mRNA expression levels. These findings and the observed inverse correlation between Grin $2 b$ mRNA expression and methylation at rCpG1-3 is a strong indication that methylation at this region is important for transcriptional regulation and responsive to early life BPA exposures in females. Notably, rCpGs 1-3 are part of a predicted binding site for Nrf1, which has been shown to regulate Grin $2 b$ expression $^{27}$ and to be sensitive to DNA methylation ${ }^{28,35,36}$. Thus it is possible that the correlation between methylation at these CpGs and Grin2b mRNA expression in females is due to diminished Nrf1 binding. Nrf1 is not the only TF predicted to bind to this region, but the only one with a documented link to Grin $2 b$ and brain development and a binding site that is conserved between rat and human (see Supplementary Table S3).

In the human samples, we investigated DNA methylation at a homologous region in the GRIN2B gene. The analysed CpGs lie in a CpG island and in the only region nearby the GRIN2B gene that is predicted to be a strong promoter, based on data sets relevant for transcriptional regulation produced in the ENCODE project ${ }^{37}$ (see Supplementary Fig. S2). In the human data, we also observed a sexual dimorphic link where prenatal BPA exposure was associated to GRIN2B methylation in 7-year old girls, but not in boys of the same age. However, in humans, higher prenatal BPA exposure was associated with higher methylation levels.

The fact that we found opposite associations between BPA exposure and Grin $2 b$ methylation levels in humans compared to rats could be due to several reasons. Firstly, this could reflect differences between species, similarly to what was found in a previous study comparing DNA methylation of repetitive elements between humans and 
a

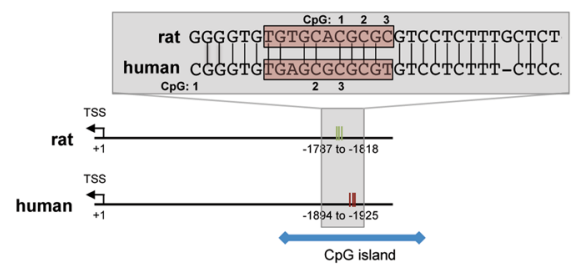

b
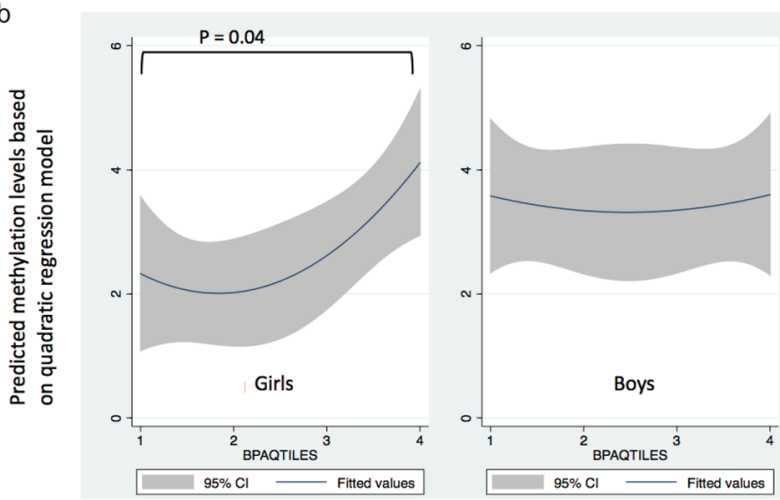

C

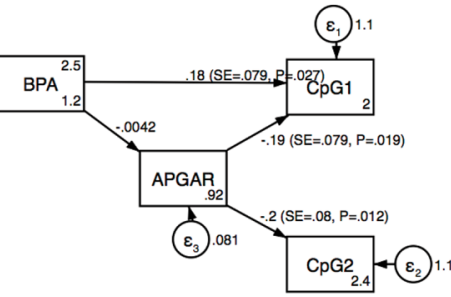

Figure 2. Relationship between GRIN2B methylation patterns and prenatal BPA levels. (a) Genomic location and sequence of the region analysed for DNA methylation relative to the transcriptional start site (TSS) of rat and human GRIN2B. The predicted Nrf1 binding site is marked with a red box. Despite lying in a well-conserved region, only rat (r) CpG 1 (corresponding to human (h) CpG 3) is conserved between the two species. In human, the analysed region is part of a CpG island. (b) Sex-dependent correlations between methylation cluster at the first CpG site, using the quadratic regression model. (c) Structure equation model (SEM) showing how prenatal BPA levels and APGAR scores at the $5^{\text {th }}$ minute could jointly influence methylations at two CpG sites simultaneously.

mice ${ }^{38}$. Of note, only rat (r)CpG1 corresponding to human (h) CpG3, is conserved between humans and rats. Human CpG1 (where associations to BPA levels were found) is not conserved between primates and rodents (Fig. 1b). Thus, the methylation changes, despite affecting a homologous region, were not found at the exact same CpGs. Secondly, the DNA methylation analyses were not conducted at the same age in human subjects and in the animals. While the rats were sacrificed at adulthood (52 weeks of age), the human samples were taken from 7-year old children. It is known that the NMDA receptor subunit $2 \mathrm{~b}$, which is encoded by Grin $2 b$, is predominantly expressed during late prenatal and early postnatal development and replaced by another subunit later on in life, which changes the signalling properties of the receptor ${ }^{26,39}$. Therefore, the observed increase in Grin $2 b$ expression in the adult rats could disturb the composition of the NMDA receptors and lead to an altered signalling function. If a similar correlation between methylation and expression of Grin $2 b$ exists in humans, we might speculate that BPA exposure leads to decreased Grin $2 b$ levels during early life where the protein encoded by this gene has important functions. Thirdly, even though we are focusing on prenatal exposure for BPA as a single chemical in the human data, it should be kept in mind that real life exposure is a matter of complicated mixtures. For example, we have shown that several alkyl phenols (including BPA) and more than 10 phthalate metabolites were all identified in prenatal urine of more than 2,300 pregnant women enrolled in the SELMA study during the period $2007-2010^{40}$. In addition, exposures to these compounds are often correlated ${ }^{41}$. In contrast, in experimental animal experiments, other exposures can be largely excluded and controlled for. Thus, while in the animals changes in Grin $2 b$ methylation can be truly assigned to BPA exposure only, the associations observed in humans are most likely due to mixed exposures.

Furthermore, the DNA methylation analyses were performed in different tissues, and there is limited data on the correlations between brain and buccal DNA methylation patterns. Nevertheless, a number of studies have addressed the relationship between DNA methylation patterns in blood and different brain areas, and there are public resources, such as the Blood Brain DNA Methylation Comparison Tool (ref..$^{42}$ based on the data by Hannon et al. ${ }^{43}$ ) where correlations between these two tissues can be interrogated. Using this tool we found that hCpG1 methylation is significantly correlated between blood and 2 out of 4 assessed brain areas: prefrontal cortex and superior temporal gyrus (see Supplementary Fig. S2), based on around 70 individuals. Further, employing data from other published sources ${ }^{44,45}$, we found a significant positive correlation between blood and saliva methylation at hCpG1 (based on 17 individuals) (see Supplementary Fig. S2). No correlation was found for blood/ saliva and buccal epithelial, however, we could only identify one study based on 5 individuals addressing this relationship ${ }^{45}$. Taken together, the available data suggest that the methylation at hCpG1 correlates between different tissues, implicating that the increased methylation at hCpG1 found in buccal epithelial of higher exposed girls could reflects an increase at this site in the target tissue (brain). 
Our finding of sex-dependent effects of BPA on the DNA methylation pattern of Grin $2 b$, observed in both human and animal data, are in line with previous studies in humans showing sexual-dimorphic effects of BPA on both DNA methylation ${ }^{9}$ and neurodevelopmental outcomes ${ }^{10}$. Human data is supported by findings in rodents, suggesting that persistent changes in brain functions by early life BPA exposure are sex-specific, often blunting or abolishing natural sexual dimorphisms $\mathrm{s}^{12,31,46,47}$. Together, these findings emphasize the importance of investigating the impact of BPA and other EDCs on the two sexes separately.

We also found that the APGAR score was associated with methylation levels at hCpG1 and hCpG2 within the GRIN2B gene. Low APGAR scores might reflect hypoxic events occurring in the uterus or the process of delivery, and hypoxia has been found to lead to global hypermethylation in some tissues ${ }^{48-50}$. Furthermore, in a genome-wide methylation analysis, methylation levels at specific sites were directly associated with the length of birth, i.e. duration of hypoxic condition ${ }^{51}$. Most prior clinical epigenetic studies have treated APGAR scores as a covariate. However, APGAR scores at the fifth minute have been found to predict long-term neurological disabilities and cognitive functions $s^{52}$ and the risk for neurodevelopmental disorders, such as ADHD ${ }^{53}$ and $\mathrm{ASD}^{54}$. Interestingly, several studies have reported associations between genetic variants in the GRIN2B gene and increased risks for neurodevelopmental disorders ${ }^{24}$. It remains to be determined if methylation of GRIN2B is also a predictor for such risks or if it is even underlying associations between hypoxic events or BPA exposure and neurodevelopmental impairments.

Limitations. One limitation of this study is the lack of behavioural assays to evaluate the BPA effect on the phenotype in the same rats as the changes in Grin $2 b$ methylation and expression were measured. Nevertheless, the literature suggests that BPA exposure in similar experimental settings leads to changes in behaviour and brain function $^{12,31,46,47}$.

Additionally the study does not address molecular events leading to the observed DNA methylation changes upon BPA exposure. Published results show that BPA can affect enzymes involved in the regulation of DNA methylation in rodent brain, such as the level of Dnmt $1^{55}$ and localization of Tet $2^{56}$. Additionally, we have shown that BPA affects DNA methylation of specific genes via ER $\beta^{21,57}$, by recruitment of thymine DNA glycosylase, an enzyme involved in DNA demethylation, to specific genomic loci ${ }^{57}$. These are all possible mechanisms underlying the findings presented here. Potentially, effects on Grin $2 b$ expression and regulation could also be results of other organismal changes, such as metabolic alterations, induced by the exposure rather than direct actions of BPA on DNA methylation regulation. Anyhow, by adjusting for growth parameters, we could exclude their involvement on the observed differences on Grin $2 b$.

Furthermore, the inaccessibility of brain tissues in humans forced us to measure DNA methylation in a peripheral tissue (i.e., buccal epithelium). However, our analyses using published data (see above) suggest that methylation at hCpG1 in the GRIN2B gene is correlated across different tissues.

Finally, this study has not addressed the functional implications of the DNA methylation changes in humans, neither its effect on gene expression nor its association to neurodevelopmental health outcomes. Nevertheless, the current findings provide a basis for further research on the link between prenatal BPA exposure, DNA methylation patterns in the GRIN2B gene, and neurodevelopment outcomes.

\section{Conclusion}

By combining an animal model and human based epidemiologic data we show here that developmental BPA exposure may influence methylation patterns at a regulatory region of the Grin $2 b$ gene. In both rats and humans, the effect of early life BPA exposure on Grin $2 b$ methylation was only seen in females, however, there were species-specific differences in location and direction of the methylation changes. In humans, GRIN2B methylation was also associated with a low APGAR score, which has been documented as a risk factor for neurodevelopmental diseases. Future research is warranted to clarify if the changes in GRIN2B methylation reported in the present study can be linked to impaired neurodevelopment or behavioural deficits associated with prenatal BPA exposure or poorer APGAR scores.

\section{Material and Methods}

Animal treatment. Animal housing and treatment are described elsewhere ${ }^{58,59}$. Briefly, female F344/DuCrl rats (Charles River) arrived at 7 weeks of age and were randomly distributed into three dosing groups (vehicle, 0.5 or $50 \mu \mathrm{g} \mathrm{BPA} / \mathrm{kg} \mathrm{BW} / \mathrm{d}$ ) and exposed to BPA via their drinking water, corresponding to approximately 0.5 or $50 \mu \mathrm{g} / \mathrm{kg}$ bw/day or vehicle control from gestational day 3.5 until weaning (postnatal day 21) using BPA (Sigma Aldrich, Missouri, USA, CAS $80-05-7$ ) with $\geq 99 \%$ purity dissolved in ethanol ( $1 \%$ of final solution). The higher dose was chosen to match the tolerable daily intake set by the U.S. FDA $(50 \mu \mathrm{g} / \mathrm{kg} / \mathrm{day})$, the lower dose approximately matches the highest estimated dietary intake of BPA in breast fed infants at the age of 0-6 month, which may reach $0.44-0.6 \mu \mathrm{g} / \mathrm{kg}$ bw/day, and the one of women of child bearing age, which can be up to $0.39 \mu \mathrm{g} / \mathrm{kg} \mathrm{bw} /$ day $^{60}$. Glass water bottles were used to minimize the background BPA exposure. Actual BPA intake and number of animals per treatment groups (previously published in Lejonklou, et al.$^{58}$ ) is provided in Supplementary Table S4. The offspring were sacrificed at the age of 52 weeks, a time point chosen well after the dosing had been completed and the rats had reached sexual maturity, to investigate truly persistent changes after the chemical exposure had long ceased. The offspring were weighted at weaning and before sacrifice at 52 weeks of age (see Supplemental Table S1). Hippocampi were dissected from a total number of 56 rats, 28 males and 28 females with 8 animals in each BPA treatment and 12 in the control group and stored in RNAlater RNA stabilization reagent (Qiagen, Hilden, Germany) to prevent RNA degradation. The study was carried out with the approval of the Uppsala ethical committee on Animal Research (ID \# C26:13) and the guidelines laid down by the European Union Legislation were followed. 


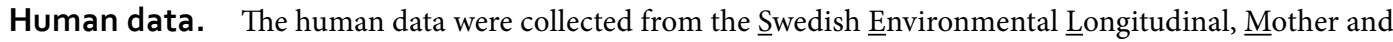
child, Asthma and allergy (SELMA) study, a pregnancy cohort study designed to investigate early life exposure to environmental chemicals and health outcomes related to growth, developmental and chronic diseases for the children. SELMA recruited pregnant women in the county of Värmland, Sweden between September 2007 and March 2010. Women who could read Swedish and were not planning to move out of the county were recruited at their first antenatal care visit; 8,394 pregnant women were identified, 6,658 were eligible and 2,582 (39\%) agreed to participate. Detailed recruitment selection criteria and sample collection procedures have been published previously $^{34}$. For the current study we used data from 318 randomly selected children at age 7 years, for which creatinine adjusted BPA levels had been measured in the $1^{\text {st }}$ trimester urine of their mothers, and oral swab samples and data on covariates had been collected for epigenetic analyses and statistical modelling, respectively. BPA levels were measured in its conjugated form in urine because urine levels are considerably higher than the ones in serum. This has two advantages: firstly, these measurements have been shown to be less sensitive to background contamination ${ }^{61-63}$ and secondly, it is more likely to highlight the between-individual variation.

First morning void urine samples were obtained from the 318 pregnant women in week 3-27 of pregnancy (median week 10, and 96\% of the samples were taken before week 13) at enrolment to the study ${ }^{34}$. Urine samples were collected in supplied glass containers at home and transferred into polypropylene tubes without any other assisting equipment for easy transportation. Samples were stored at $-20^{\circ} \mathrm{C}$ before being processed at the laboratory at division of occupational and environmental medicine, Lund University, Sweden ${ }^{64}$. Quantitative analysis of urinary BPA concentrations was conducted using a triple quadrupole linear ion trap mass spectrometry (QTRAP 5500; AB Sciex, Foster City, CA, USA) coupled to a liquid chromatography system (UFLCXR, Shimadzu Corporation, Kyoto, Japan; LC/MS/MS). The samples were prepared according to the method presented in Bornehag, et al. ${ }^{34}$ and analysed according to a method described by Berge, et al. ${ }^{65}$. Urinary creatinine concentrations were analysed according to an enzymatic method described by Mazzachi, et al. ${ }^{66}$.

Blood samples were taken from the pregnant women at the time of enrolment and the serum aliquots were stored at $-80^{\circ} \mathrm{C}$ in a biobank. For cotinine levels, a biomarker for tobacco smoke exposure, prenatal serum samples were analysed for cotinine using LC-MS/MS as a biomarker for nicotine exposure, and used to assess smoking status. A detailed description of the method is presented in Lindh, et al ${ }^{67}$. If cotinine levels were below $0.2 \mathrm{ng} / \mathrm{mL}$, subjects were categorized as non-smokers; if cotinine levels were greater than $15 \mathrm{ng} / \mathrm{mL}$, subjects were considered as active smokers; while in between $(0.2-15 \mathrm{ng} / \mathrm{mL})$, subjects were considered as passive smokers $^{68}$.

Self-administered questionnaires were collected for background information including stressful events during pregnancy. We measured the maternal stress level using the items from one of our SELMA questionnaires. The participant was asked to answer if the mother had experienced at least one of the following situations during the pregnancy: (a) unemployment of the participant or the partner/spouse, (b) severe disease or injury in the participant, (c) severe disease or injury in the partner/spouse, children, sibling, or parents, (d) death of partner/spouse, children, sibling, or parents, (e) separation/divorce or any crisis in the relationship, and (f) financial crisis for the participant or the partner/spouse. Any situation at any time point during the pregnancy was coded as maternal stressful event.

Data on APGAR scores at the fifth minute for the child were imported from the Swedish National Birth Register.

The SELMA study is approved by Ethical Board of Uppsala, Sweden (2015-06-10, Dnr: 2015/177). Informed consents were obtained from all adult participants and guardians of under age participants. All human experimental methods and procedures were performed in accordance with the relevant ethical guidelines and regulations.

Gene expression analysis. Extraction of total RNA from rat hippocampi was carried using the AllPrep ${ }^{\circledR}$ DNA/RNA/Protein Mini Kit (Qiagen, Hilden, Germany) in accordance with the manufacturer's instructions. Quantity and quality of isolated RNA was assessed using a (TECAN, Grödig, Austria). Due to insufficient quality, 4 samples (1 male and 3 females) had to be excluded from the analysis. One $\mu \mathrm{g}$ of total RNA was treated with DNAseI (New England Biolabs, Ipswich, USA) and reverse transcribed using iScript ${ }^{\mathrm{TM}}$ Reverse Transcription Supermix (Bio-Rad Laboratories, Hercules, USA) according to manufacturer's protocol. One $\mu$ l of the resulting cDNA per reaction was used for real-time PCR using Rotor-Gene SYBR Green PCR kit on a Rotor-Gene 3000 (Qiagen, Hilden, Germany). Expression of 36B4 was used as reference gene and relative expression was calculated based on the delta-delta $\mathrm{C}_{\mathrm{T}}$ method. Primers used for real-time PCR analyses are listed in Supplementary Table S5.

DNA methylation analysis. Extraction of genomic DNA from rat hippocampi was carried out using the AllPrep ${ }^{\circledR}$ DNA/RNA/Protein Mini Kit (Qiagen, Hilden, Germany) in accordance with the manufacturer’s instructions. For human samples, isolation of genomic DNA from oral swabs was performed using BuccalAmp ${ }^{\mathrm{TM}}$ DNA extraction kit (Epicentre, Chicago, USA) according to manufacturer protocol. For 9 samples (4 males and 5 females), the DNA yield was insufficient for continued analyses. Bisulfite treatment was performed on 200 to $500 \mathrm{ng}$ of extracted genomic DNA using the EZ DNA Methylation kit (Zymo Research, Irvine, USA). One $\mu$ l of converted DNA was used for PCR amplification, and the PCR product was sequenced by pyrosequencing on a Pyromark Q24 (Qiagen, Hilden, Germany) in accordance with the manufacturer's protocol, and the PyroMark Q24 software (Qiagen Pyromark Q24, version 5.0) was used for calculating percentage methylation at the analysed CpGs. To test the reliability of the assays, standard curves were performed using commercial genomic DNA standards (Qiagen, Hilden, Germany) (Supplementary Fig. S3). Primers used for pyrosequencing analyses are listed in Table S5.

Statistical and bioinformatics analysis. For animal data, two-way ANOVA was used to compare level of gene expression between-groups and one-way ANOVA for comparing methylation levels within each group. The results were corrected using the Tukey's post-hoc test. To adjust for possible batch effects or outliers in the DNA 
methylation measurements, we used rank-based inverse normal transformation to reprocess the raw data and re-analyze the effect of BPA exposure on methylation levels. We further used generalized linear model to regress methylation levels against both BPA exposure status and body weight change in order to control for growth rate. For correlation between DNA methylation and mRNA expression, Pearson analysis was used. The number of independent data points $(\mathrm{n})$ is indicated in the figure legends of the corresponding graphs. Data is shown as mean + standard deviation (SD). The level of significance was selected at $\mathrm{P}<0.05$.

For human data, we implemented a multi-step statistical strategy to analyse the relationship between prenatal BPA exposure and methylations at GRIN2B at the age of 7 years. The steps included (1) clustering analysis to identify patterns of methylations, (2) ordered logistic regression to examine the effect of BPA exposure on methylations while adjusting for potential confounders, (3) stratified analysis by sex, and (4) structural equation models to perform multi-CpG-site analysis. The distributions of methylation levels at three CpG sites showed highly skewed-to-left distributions due to large numbers of samples showing $0 \%$ methylation. Such a skewed distribution of outcome might have caused the violation of normality assumption for residual errors in linear regression models, where methylation levels were regressed against prenatal BPA levels and other covariates. Therefore, the k-means clustering algorithm was used to identify clusters of methylation levels for each CpG site, as described in previous studies ${ }^{69,70}$. The appropriate number of clusters based on methylation levels at each CpG site was determined using the Calinski-Harabasz $(\mathrm{CH})$ criteria (see Supplementary Fig. S3). The optimal value of clusters was chosen when $\mathrm{CH}$ pseudo-F value encountered the first abrupt increase. The drastic increase in this heuristic value is often used to indicate the identification of number of distinct clusters. Similar approaches have been described in other bioinformatics studies ${ }^{71}$.

The relationship between each cluster variable for each CpG site and prenatal BPA levels (expressed as a quartile variable) was then assessed using an ordered logistic regression model adjusted for potential covariates, including maternal smoking during pregnancy (based on cotinine concentration in prenatal serum), maternal self-reported stressful events during pregnancy (based on a retrospective questionnaire post partum), and APGAR scores (measured at birth at the fifth minute). Potential confounders/mediators were selected from pair-wise correlation tests (Spearman correlation test $\mathrm{p}$-value $<0.05$ ) or from previous evidence for their impacts on epigenetic patterns ${ }^{72,73}$. No covariate was found to remarkably impact the effect size of the key predictor, i.e. BPA levels (i.e., changing greater than $10 \%$ of the original regression coefficient).

Two-side alpha value $=0.05$ was used to determine which $\mathrm{CpG}$ methylation levels could be predicted by the level of prenatal BPA. Finally, significant contributors were selected for methylation levels at each CpG site, and structural equation models (SEM) were used to examine the joint impact of predictors (with a focus on prenatal BPA levels) on multi-site methylation levels. The purpose of using SEM was to assess the relationship among multiple predictors and multiple outcomes simultaneously. The final SEM model was selected based on the Root Mean Square Error of Approximation (RMSEA) value with 0.1 as the cut-off. In addition to RMSEA, we also compared the selected model with the saturated model (i.e., as many estimated parameters as data points) to avoid over-fitting.

TF binding site prediction was performed using the JASPAR database ${ }^{74}$. Input sequence for rat was: GTGTGCACGCGCGT and for human: CGGGTGTGAGCGCGCGT. The list of all identified TF binding sites is provided as Supplementary Table S3.

Data availability. The datasets generated from rats are available from the corresponding author on reasonable request. The De-identified SELMA exposure data may be available upon request in case a mutually agreed research plan and ethical approval documents are provided.

\section{References}

1. Mikolajewska, K., Stragierowicz, J. \& Gromadzinska, J. Bisphenol A - Application, sources of exposure and potential risks in infants, children and pregnant women. International journal of occupational medicine and environmental health 28, 209-241, https://doi. org/10.13075/ijomeh.1896.00343 (2015)

2. Calafat, A. M., Ye, X., Wong, L. Y., Reidy, J. A. \& Needham, L. L. Exposure of the U.S. population to bisphenol A and 4-tertiaryoctylphenol: 2003-2004. Environ Health Perspect 116, 39-44, https://doi.org/10.1289/ehp.10753 (2008).

3. WHO/FAO. Toxicological and Health Aspects of Bisphenol A. (World Health Organization, Switzerland, 2010).

4. WHO/UNEP. State of the science of endocrine disrupting chemicals (2013).

5. Diamanti-Kandarakis, E. et al. Endocrine-disrupting chemicals: an Endocrine Society scientific statement. Endocr Rev 30, 293-342, https://doi.org/10.1210/er.2009-0002 (2009).

6. Gore, A. C. et al. EDC-2: The Endocrine Society's Second Scientific Statement on Endocrine-Disrupting Chemicals. Endocr Rev 36, E1-E150, https://doi.org/10.1210/er.2015-1010 (2015).

7. Varshney, M. \& Nalvarte, I. Genes, Gender, Environment, and Novel Functions of Estrogen Receptor Beta in the Susceptibility to Neurodevelopmental Disorders. Brain sciences 7, https://doi.org/10.3390/brainsci7030024 (2017).

8. Berenbaum, S. A. \& Beltz, A. M. Sexual differentiation of human behavior: effects of prenatal and pubertal organizational hormones. Frontiers in neuroendocrinology 32, 183-200, https://doi.org/10.1016/j.yfrne.2011.03.001 (2011).

9. Rochester, J. R. Bisphenol A and human health: a review of the literature. Reprod Toxicol 42, 132-155, https://doi.org/10.1016/j. reprotox.2013.08.008 (2013).

10. Mustieles, V., Perez-Lobato, R., Olea, N. \& Fernandez, M. F. Bisphenol A: Human exposure and neurobehavior. Neurotoxicology 49, 174-184, https://doi.org/10.1016/j.neuro.2015.06.002 (2015).

11. Ejaredar, M., Lee, Y., Roberts, D. J., Sauve, R. \& Dewey, D. Bisphenol A exposure and children's behavior: A systematic review. Journal of exposure science \& environmental epidemiology, https://doi.org/10.1038/jes.2016.8 (2016).

12. Negri-Cesi, P. Bisphenol A Interaction With Brain Development and Functions. Dose Response 13, https://doi.org/10.1177/ 1559325815590394 (2015).

13. McCabe, C., Anderson, O. S., Montrose, L., Neier, K. \& Dolinoy, D. C. Sexually Dimorphic Effects of Early-Life Exposures to Endocrine Disruptors: Sex-Specific Epigenetic Reprogramming as a Potential Mechanism. Current environmental health reports 4 , 426-438, https://doi.org/10.1007/s40572-017-0170-z (2017).

14. Kundakovic, M. \& Champagne, F. A. Epigenetic perspective on the developmental effects of bisphenol A. Brain, behavior, and immunity 25, 1084-1093, https://doi.org/10.1016/j.bbi.2011.02.005 (2011). 
15. Mileva, G., Baker, S. L., Konkle, A. T. \& Bielajew, C. Bisphenol-A: epigenetic reprogramming and effects on reproduction and behavior. International journal of environmental research and public health 11, 7537-7561, https://doi.org/10.3390/ijerph110707537 (2014).

16. Jacobs, M. N., Marczylo, E. L., Guerrero-Bosagna, C. \& Rüegg, J. Marked for Life: Epigenetic Effects of Endocrine Disrupting Chemicals. Annu. Rev. Environ. Resour. 42, 105-160, https://doi.org/10.1146/annurev-environ-102016-061111 (2017).

17. Bale, T. L. Epigenetic and transgenerational reprogramming of brain development. Nat Rev Neurosci 16, 332-344, https://doi. org/10.1038/nrn3818 (2015).

18. Keverne, E. B. Significance of epigenetics for understanding brain development, brain evolution and behaviour. Neuroscience 264, 207-217, https://doi.org/10.1016/j.neuroscience.2012.11.030 (2014).

19. Marczylo, E. L., Jacobs, M. N. \& Gant, T. W. Environmentally induced epigenetic toxicity: potential public health concerns. Critical reviews in toxicology 46, 676-700, https://doi.org/10.1080/10408444.2016.1175417 (2016).

20. Xu, X. H., Zhang, J., Wang, Y. M., Ye, Y. P. \& Luo, Q. Q. Perinatal exposure to bisphenol-A impairs learning-memory by concomitant down-regulation of N-methyl-D-aspartate receptors of hippocampus in male offspring mice. Horm Behav 58, 326-333, https://doi. org/10.1016/j.yhbeh.2010.02.012 (2010).

21. Kitraki, E., Nalvarte, I., Alavian-Ghavanini, A. \& Ruegg, J. Developmental exposure to bisphenol A alters expression and DNA methylation of Fkbp5, an important regulator of the stress response. Mol Cell Endocrinol 417, 191-199, https://doi.org/10.1016/j. mce.2015.09.028 (2015).

22. Kundakovic, M. et al. DNA methylation of BDNF as a biomarker of early-life adversity. Proceedings of the National Academy of Sciences of the United States of America 112, 6807-6813, https://doi.org/10.1073/pnas.1408355111 (2015).

23. Dorval, K. M. et al. Association of the glutamate receptor subunit gene GRIN2B with attention-deficit/hyperactivity disorder. Genes Brain Behav 6, 444-452, https://doi.org/10.1111/j.1601-183X.2006.00273.x (2007)

24. Hu, C., Chen, W., Myers, S. J., Yuan, H. \& Traynelis, S. F. Human GRIN2B variants in neurodevelopmental disorders. J Pharmacol Sci 132, 115-121, https://doi.org/10.1016/j.jphs.2016.10.002 (2016).

25. Martucci, L. et al. N-methyl-D-aspartate receptor NR2B subunit gene GRIN2B in schizophrenia and bipolar disorder: Polymorphisms and mRNA levels. Schizophr Res 84, 214-221, https://doi.org/10.1016/j.schres.2006.02.001 (2006).

26. Cull-Candy, S., Brickley, S. \& Farrant, M. NMDA receptor subunits: diversity, development and disease. Curr Opin Neurobiol 11, $327-335(2001)$

27. Priya, A., Johar, K. \& Wong-Riley, M. T. Nuclear respiratory factor 2 regulates the expression of the same NMDA receptor subunit genes as NRF-1: both factors act by a concurrent and parallel mechanism to couple energy metabolism and synaptic transmission. Biochim Biophys Acta 1833, 48-58, https://doi.org/10.1016/j.bbamcr.2012.10.014 (2013).

28. Domcke, S. et al. Competition between DNA methylation and transcription factors determines binding of NRF1. Nature 528, 575-579, https://doi.org/10.1038/nature16462 (2015).

29. Marsman, A. et al. Glutamate in schizophrenia: a focused review and meta-analysis of (1)H-MRS studies. Schizophr Bull 39, 120-129, https://doi.org/10.1093/schbul/sbr069 (2013)

30. van Elst, L. T. et al. Increased prefrontal and hippocampal glutamate concentration in schizophrenia: evidence from a magnetic resonance spectroscopy study. Biol Psychiatry 58, 724-730, https://doi.org/10.1016/j.biopsych.2005.04.041 (2005).

31. Kunz, N. et al. Developmental and metabolic brain alterations in rats exposed to bisphenol A during gestation and lactation. Int $J$ Dev Neurosci 29, 37-43, https://doi.org/10.1016/j.ijdevneu.2010.09.009 (2011).

32. Monyer, H., Burnashev, N., Laurie, D. J., Sakmann, B. \& Seeburg, P. H. Developmental and regional expression in the rat brain and functional properties of four NMDA receptors. Neuron 12, 529-540 (1994).

33. Alonso-Magdalena, P., Rivera, F. J. \& Guerrero-Bosagna, C. Bisphenol-A and metabolic diseases: epigenetic, developmental and transgenerational basis. Environ Epigenet 2, dvw022, https://doi.org/10.1093/eep/dvw022 (2016).

34. Bornehag, C. G. et al. The SELMA study: a birth cohort study in Sweden following more than 2000 mother-child pairs. Paediatr Perinat Epidemiol 26, 456-467, https://doi.org/10.1111/j.1365-3016.2012.01314.x (2012).

35. Choi, Y. S., Kim, S., Kyu Lee, H., Lee, K. U. \& Pak, Y. K. In vitro methylation of nuclear respiratory factor-1 binding site suppresses the promoter activity of mitochondrial transcription factor A. Biochem Biophys Res Commun 314, 118-122 (2004).

36. Wang, J. et al. NRF1 coordinates with DNA methylation to regulate spermatogenesis. FASEB J 31, 4959-4970, https://doi. org/10.1096/f.201700093R (2017).

37. Karolchik, D. et al. The UCSC Genome Browser database: 2014 update. Nucleic acids research 42, D764-770, https://doi.org/10.1093/ nar/gkt1168 (2014).

38. Faulk, C. et al. Detection of differential DNA methylation in repetitive DNA of mice and humans perinatally exposed to bisphenol A. Epigenetics 11, 489-500, https://doi.org/10.1080/15592294.2016.1183856 (2016).

39. Jantzie, L. L. et al. Developmental expression of N-methyl-D-aspartate (NMDA) receptor subunits in human white and gray matter: potential mechanism of increased vulnerability in the immature brain. Cereb Cortex 25, 482-495, https://doi.org/10.1093/cercor/ bht246 (2015).

40. Shu, H. et al. Temporal Trends of Phthalate Exposures during 2007-2010 in Swedish Pregnant Women (2017).

41. Frederiksen, H., Jorgensen, N. \& Andersson, A. M. Correlations between phthalate metabolites in urine, serum, and seminal plasma from young Danish men determined by isotope dilution liquid chromatography tandem mass spectrometry. J Anal Toxicol 34, $400-410(2010)$.

42. Eilis Hannon, K. L., Schalkwyk, L. \& Mill, J. Blood Brain DNA Methylation Comparison Tool, http://epigenetics.iop.kcl.ac.uk/ bloodbrain/ (2017).

43. Hannon, E., Lunnon, K., Schalkwyk, L. \& Mill, J. Interindividual methylomic variation across blood, cortex, and cerebellum: implications for epigenetic studies of neurological and neuropsychiatric phenotypes. Epigenetics 10, 1024-1032, https://doi.org/10. 1080/15592294.2015.1100786 (2015).

44. Langie, S. A. et al. Whole-Genome Saliva and Blood DNA Methylation Profiling in Individuals with a Respiratory Allergy. PLoS One 11, e0151109, https://doi.org/10.1371/journal.pone.0151109 (2016).

45. Slieker, R. C. et al. Identification and systematic annotation of tissue-specific differentially methylated regions using the Illumina 450k array. Epigenetics \& chromatin 6, 26, https://doi.org/10.1186/1756-8935-6-26 (2013).

46. Gioiosa, L., Palanza, P., Parmigiani, S. \& Vom Saal, F. S. Risk Evaluation of Endocrine-Disrupting Chemicals: Effects of Developmental Exposure to Low Doses of Bisphenol A on Behavior and Physiology in Mice (Mus musculus). Dose Response 13, https://doi.org/10.1177/1559325815610760 (2015).

47. Wolstenholme, J. T., Rissman, E. F. \& Connelly, J. J. The role of Bisphenol A in shaping the brain, epigenome and behavior. Horm Behav 59, 296-305, https://doi.org/10.1016/j.yhbeh.2010.10.001 (2011).

48. Robinson, C. M., Neary, R., Levendale, A., Watson, C. J. \& Baugh, J. A. Hypoxia-induced DNA hypermethylation in human pulmonary fibroblasts is associated with Thy-1 promoter methylation and the development of a pro-fibrotic phenotype. Respir Res 13, 74, https://doi.org/10.1186/1465-9921-13-74 (2012).

49. Xu, X. et al. Hypoxia-induced endothelial-mesenchymal transition is associated with RASAL1 promoter hypermethylation in human coronary endothelial cells. FEBS Lett 590, 1222-1233, https://doi.org/10.1002/1873-3468.12158 (2016).

50. Yuen, R. K., Chen, B., Blair, J. D., Robinson, W. P. \& Nelson, D. M. Hypoxia alters the epigenetic profile in cultured human placental trophoblasts. Epigenetics 8, 192-202, https://doi.org/10.4161/epi.23400 (2013). 
51. Almgren, M. et al. Cesarean delivery and hematopoietic stem cell epigenetics in the newborn infant: implications for future health? American journal of obstetrics and gynecology 211, 502 e501-508, https://doi.org/10.1016/j.ajog.2014.05.014 (2014).

52. Ehrenstein, V. et al. Association of Apgar score at five minutes with long-term neurologic disability and cognitive function in a prevalence study of Danish conscripts. BMC Pregnancy Childbirth 9, 14, https://doi.org/10.1186/1471-2393-9-14 (2009).

53. Li, J., Olsen, J., Vestergaard, M. \& Obel, C. Low Apgar scores and risk of childhood attention deficit hyperactivity disorder. J Pediatr 158, 775-779, https://doi.org/10.1016/j.jpeds.2010.10.041 (2011).

54. Polo-Kantola, P. et al. Obstetric risk factors and autism spectrum disorders in Finland. J Pediatr 164, 358-365, https://doi. org/10.1016/j.jpeds.2013.09.044 (2014).

55. Zhou, R., Chen, F., Chang, F., Bai, Y. \& Chen, L. Persistent overexpression of DNA methyltransferase 1 attenuating GABAergic inhibition in basolateral amygdala accounts for anxiety in rat offspring exposed perinatally to low-dose bisphenol A. Journal of psychiatric research 47, 1535-1544, https://doi.org/10.1016/j.jpsychires.2013.05.013 (2013).

56. Kurian, J. R. et al. The Methylcytosine Dioxygenase Ten-Eleven Translocase-2 (tet2) Enables Elevated GnRH Gene Expression and Maintenance of Male Reproductive Function. Endocrinology 157, 3588-3603, https://doi.org/10.1210/en.2016-1087 (2016).

57. Liu, Y. et al. Oestrogen receptor beta regulates epigenetic patterns at specific genomic loci through interaction with thymine DNA glycosylase. Epigenetics \& chromatin 9, 7, https://doi.org/10.1186/s13072-016-0055-7 (2016).

58. Lejonklou, M. H. et al. Effects of Low-Dose Developmental Bisphenol A Exposure on Metabolic Parameters and Gene Expression in Male and Female Fischer 344 Rat Offspring. Environ Health Perspect 125, 067018, https://doi.org/10.1289/EHP505 (2017).

59. Lind, T. et al. Low-dose developmental exposure to bisphenol A induces sex-specific effects in bone of Fischer 344 rat offspring. Environ Res 159, 61-68, https://doi.org/10.1016/j.envres.2017.07.020 (2017).

60. EFSA. Scientific opinion about bisphenol A. https://www.efsa.europa.eu/sites/default/files/corporate_publications/files/ factsheetbpa150121.pdf (2015).

61. Vandenberg, L. N., Hauser, R., Marcus, M., Olea, N. \& Welshons, W. V. Human exposure to bisphenol A (BPA). Reprod Toxicol 24, 139-177, https://doi.org/10.1016/j.reprotox.2007.07.010 (2007).

62. Churchwell, M. I. et al. Comparison of life-stage-dependent internal dosimetry for bisphenol A, ethinyl estradiol, a reference estrogen, and endogenous estradiol to test an estrogenic mode of action in Sprague Dawley rats. Toxicol Sci 139, 4-20, https://doi. org/10.1093/toxsci/kfu021 (2014)

63. Calafat, A. M. et al. Misuse of blood serum to assess exposure to bisphenol A and phthalates. Breast Cancer Res 15, 403, https://doi. org/10.1186/bcr3494 (2013).

64. Bornehag, C. G. et al. Prenatal phthalate exposures and anogenital distance in Swedish boys. Environmental health perspectives 123, 101-107, https://doi.org/10.1289/ehp.1408163 (2015).

65. Berge, T. L., Lygre, G. B., Jonsson, B. A., Lindh, C. H. \& Bjorkman, L. Bisphenol A concentration in human saliva related to dental polymer-based fillings. Clin Oral Investig, https://doi.org/10.1007/s00784-017-2055-9 (2017).

66. Mazzachi, B. C., Peake, M. J. \& Ehrhardt, V. Reference range and method comparison studies for enzymatic and Jaffe creatinine assays in plasma and serum and early morning urine. Clinical laboratory 46, 53-55 (2000).

67. Lindh, C. H. et al. Blood serum concentrations of perfluorinated compounds in men from Greenlandic Inuit and European populations. Chemosphere 88, 1269-1275, https://doi.org/10.1016/j.chemosphere.2012.03.049 (2012).

68. Jefferis, B. J. et al. Cotinine-assessed second-hand smoke exposure and risk of cardiovascular disease in older adults. Heart 96, 854 (2010).

69. Liu, L. et al. Decomposition of RNA methylome reveals co-methylation patterns induced by latent enzymatic regulators of the epitranscriptome. Mol Biosyst 11, 262-274, https://doi.org/10.1039/c4mb00604f (2015).

70. Tsuji, S. et al. Network-based analysis for identification of candidate genes for colorectal cancer progression. Biochem Biophys Res Commun 476, 534-540, https://doi.org/10.1016/j.bbrc.2016.05.158 (2016).

71. Lord, E., Diallo, A. B. \& Makarenkov, V. Classification of bioinformatics workflows using weighted versions of partitioning and hierarchical clustering algorithms. BMC Bioinformatics 16, 68, https://doi.org/10.1186/s12859-015-0508-1 (2015).

72. Montalvo-Ortiz, J. L. et al. The role of genes involved in stress, neural plasticity, and brain circuitry in depressive phenotypes: Convergent findings in a mouse model of neglect. Behav Brain Res 315, 71-74, https://doi.org/10.1016/j.bbr.2016.08.010 (2016).

73. Satta, R. et al. Nicotine decreases DNA methyltransferase 1 expression and glutamic acid decarboxylase 67 promoter methylation in GABAergic interneurons. Proceedings of the National Academy of Sciences of the United States of America 105, 16356-16361, https:// doi.org/10.1073/pnas.0808699105 (2008).

74. Khan, A. et al. JASPAR 2018: update of the open-access database of transcription factor binding profiles and its web framework. Nucleic Acids Res 46, D260-D266, https://doi.org/10.1093/nar/gkx1126 (2018).

\section{Acknowledgements}

We would like to thank Dr. Erik Lampa for his assistance and input for the statistical analyses. This research was funded by the European Union's Horizon 2020 research and innovation programme under grant agreement No 634880, EDC-MixRisk (PL, SRR, CGB, DL, JR), the Swedish Research Council Formas No 216-2012-475 (PML), 210-2012-1502 (JR) and 216-2013-1966 (AAG, SRR, JR).

\section{Author Contributions}

A.A.G., P.L., C.G.B. and J.R. conceived and designed this study. P.M.L., M.H.L. and L.D. performed the animal work and provided the rat brains. A.A.G., and M.T. conducted analyses in the rat brains. C.L. performed the chemical analyses in the urine samples of the SELMA mothers. S.R. analysed DNA methylation in buccal samples of the SELMA children and, together with P.L. and C.G.B. performed the biostatistical analyses for the human cohort. A.A.G., P.L., C.G.B. and J.R. wrote, revised and finalized the manuscript. All authors reviewed and approved the final manuscript.

\section{Additional Information}

Supplementary information accompanies this paper at https://doi.org/10.1038/s41598-018-29732-9.

Competing Interests: The authors declare no competing interests.

Publisher's note: Springer Nature remains neutral with regard to jurisdictional claims in published maps and institutional affiliations. 
(i) Open Access This article is licensed under a Creative Commons Attribution 4.0 International License, which permits use, sharing, adaptation, distribution and reproduction in any medium or format, as long as you give appropriate credit to the original author(s) and the source, provide a link to the Creative Commons license, and indicate if changes were made. The images or other third party material in this article are included in the article's Creative Commons license, unless indicated otherwise in a credit line to the material. If material is not included in the article's Creative Commons license and your intended use is not permitted by statutory regulation or exceeds the permitted use, you will need to obtain permission directly from the copyright holder. To view a copy of this license, visit http://creativecommons.org/licenses/by/4.0/.

(C) The Author(s) 2018 